\title{
CREENCIAS SOBRE LA NATURALEZA DE LA CIENCIA. UN ESTUDIO CON TITULADOS UNIVERSITARIOS EN FORMACIÓN INICIAL PARA SER PROFESORES DE EDUCACIÓN SECUNDARIA
}

\author{
José Antonio Acevedo Díaz \\ Consejería de Educación de la Junta de Andalucía, España \\ Pilar Acevedo Romero \\ Universidad de Sevilla, España
}

\section{LA NATURALEZA DE LA CIENCIA COMO OBJETIVO PRIORITARIO DE LA ENSEÑANZA DE LAS CIENCIAS}

Las concepciones epistemológicas y sociológicas sobre la naturaleza de la ciencia y acerca de cómo se elabora el conocimiento científico condicionan las finalidades y los objetivos de la educación científica, lo que a su vez dota de distintos significados al principio de comprensividad y equidad conocido como alfabetización científica y tecnológica para todas las personas (science \& tec hnolgy literacy —STL_, and science \& technology for allSTA-), que en los últimos años se propugna con asiduidad en los informes de política educativa de numerosos países y organismos internacionales relacionados con la educación, así como en los de toma de posición de destacadas asociaciones profesionales de profesores de ciencias e investigadores en didáctica de las ciencias. También se asume que uno de los objetivos más importantes de la enseñanza obligatoria de las ciencias es que los estudiantes de educación secundaria lleguen a adquirir una mejor comprensión de la naturaleza de la ciencia (Matthews, 1994a; Meichtry, 1993). Aunque, como hace notar Lederman (1992), el significado de este viejo objetivo educativo ha ido cambiando con el tiempo, en los últimos años ha aparecido como uno de los componentes esenciales de la alfabetización científica y tecnológica para todas las personas (Reid y Hodson, 1989) y de la educación CTS -Ciencia, Tecnología y Sociedad- (Acevedo, 1994, 1996; Manassero, Vázquez y Acevedo, 2001a; Vázquez, 1999; Vázquez y Manassero, 1995a). En este sentido, Hodson (1994) sostiene que uno de los tres principales objetivos de la enseñanza de las ciencias debe ser el aprendizaje sobre la naturaleza de la ciencia con el fin de desarrollar una mejor comprensión de la misma y de sus métodos, así como contribuir a tomar conciencia de las interacciones entre ciencia, tecnología y sociedad. Por último, hay que destacar también que las reformas de la enseñanza de las ciencias emprendidas en la década de los 90 suelen incorporar este objetivo de manera explícita, como ha ocurrido en los EE.UU., por ejemplo, con las llevadas a cabo por la prestigiosa American Association for the Advancement of Science (AAAS, 1990, 1993) y por el National Research Council(NRC,1996).

\section{LA NATURALEZA DE LA CIENCIA EN LA INVESTIGACIÓN DE LA DIDÁCTICA DE LAS CIENCIAS}

El interés que acabamos de señalar por la comprensión de la naturaleza de la ciencia y el desarrollo del conocimiento científico ha originado un important e campo de investigación didáctica, con amplia tradición en la actualidad, que se caracteriza por distintas líneas de investigación relacionadas entre sí. Según Lederman (1992) éstas son:

- La evaluación de las concepciones de los estudiantes sobre la naturaleza de la ciencia.

- La elaboración, utilización y evaluación de currículo destinados a mejorar estas concepciones. 
- La evaluación de las concepciones de los profesores sobre la naturaleza de la ciencia y los esfuerzos hechos para mejorarlas.

- Las relaciones entre las concepciones del profesorado sobre la naturaleza de la ciencia, su práctica docente en el aula y las concepciones del alumnado sobre el tema.

- La elaboración de diversos instrumentos para evaluar estas concepciones y el desarrollo de las técnicas necesarias para su aplicación.

Por diversos motivos, desde la última década del siglo XX, el estudio de las concepciones del profesorado sobre la naturaleza de la ciencia se ha convertido, sin duda, en uno de los temas prioritarios de la investigación en didáctica de las ciencias, continuando vivo su interés en la actualidad. Este trabajo se ocupa de la evaluación de las creencias acerca de la naturaleza de la ciencia que tienen los futuros profesores de ciencias de educación secundaria, combinando como instrumentos de evaluación un cuestionario y la realización de entrevistas semiestructuradas. Hemos preferido usar aquí el término "creencias del profesorado" para destacar que, con frecuencia, se observa falta de reflexión explícita sobre estas cuestiones (constituyen "teorías implícitas"), hecho que ha sido expresado profusamente en la literatura sobre el tema. Así, por ejemplo, Hodson (1994) mantiene que las concepciones de los profesores sobre la ciencia se forman, generalmente de manera implícita, a pa rtir de sus propias experiencias de aprendizaje escolar y universitario, reforzándose por las imágenes estereotipadas, míticas e interesadas de la ciencia y los científicos que a menudo se transmiten en los libros de texto y materiales curriculares (Meichtry, 1993), en mucha de la divulgación científica al uso que se propaga a través de los medios de comunicación de masas (Gordon, 1984) e impregna el lenguaje cotidiano y, en general, por la llamada educación informal (Acevedo, Vázquez, Manassero y Acevedo, 2002; Manassero, Vázquez y Acevedo, 2001b). De modo similar, Fourez (1994), que las llama "filosofías espontáneas de las ciencias", sostiene que son inculcadas por el contexto cultural o por la formación científica recibida.

Desde el primer momento las in vestigaciones dirigidas a evaluar estas creencias del profesorado se han basado implícitamente en dos hipótesis: (a) que su comprensión de la naturaleza de la ciencia guarda alguna relación con la de sus estudiantes y la imagen que éstos adquieren de la ciencia y los científicos (Guilbert y Meloche, 1993; Porlán y Martín del Pozo, 1996; Porlán y Rivero, 1998; Rebollo 1998), y (b) que sus concepciones sobre el tema influyen significativamente en su forma de enseñar ciencias y en las decisiones que toman en elaula (Brickhouse, 1990; Carrascosa, Fernández, Gil y Orozco, 1993; Guilbert y Meloche, 1993; Hodson, 1994; Porlán, 1994; Porlán y Martín del Pozo, 1996; Porlán y Rivero, 1998; Porlán, Rivero y Martín del Pozo, 1998). Hipótesis que, en su día, fueron asumidas explícitamente en la introducción al currículum del área de Ciencias de la Naturaleza de la Educación Secundaria Obligatoria de la Comunidad Autónoma de Andalucía (CECJA, 1992), correpondiente a la reforma española emanada de la LOGSE:

Existe una estrecha relación entre la concepción que sobre la naturaleza de la ciencia se posee y los tipos de aprendizajes que se promueven en los alumnos." [Decreto 106/1992, anexo II, p. 4126. (CECJA, 1992)]. 
Sin embargo, por muy atractivas que sean y hasta aparentemente obvias, estas hipótesis no están suficientemente avaladas por la investigación, puesto que los resultados disponibles no son inequívocos sino más bien contradictorios y hasta contrarios (Aikenhead, 1997; Duschl y Wright, 1989; Lederman, 1992, 1999).

Además de la excelente revisión de Lederman (1992), la preparada por Koulaidis y Ogborn (1995) y la más reciente de Abd-El-Khalic y Lederman (2000), disponemos en nuestro país de las realizadas por Porlán y Rivero (1998; Porlán, Rivero y Martín del Pozo, 1998) y por Mellado (1995, 1996, 1997, 1998). A nuestro juicio, la de este último autor tiene el interés añadido de que, sin perder de vista las principales conclusiones de los trabajos extranjeros más importantes, considera ampliamente también los publicados en diversas revistas españolas, lo que supone la aproximación a nuestra realidad educativa. Del conjunto de sus publicaciones puede extraerse resumidamente que:

- $\quad$ Aunque la mayoría de las investigaciones realizadas colocan a los profesores de ciencias en alguna de las formas del positivismo, hay otras que muestran una situación más compleja de lo previsto.

- La mayor parte de las investigaciones realizadas emplean cuestionarios escritos. Es necesario utilizar otras metodologías que ayuden a profundizar más en el tema, tales como entrevistas, observaciones, etc.

- Hay casi unanimidad en señalar que estas cuestiones no son prácticamente tratadas en la formación del profesorado de ciencias, y que deberían abordarse para ayudarle a reflexionar sobre sus propias concepciones acerca de la naturaleza de la ciencia.

- No todos los trabajos muestran una clara relación entre las concepciones de los profesores sobre la naturaleza de la ciencia y las que tienen acerca de la enseñanza y el aprendizaje de las ciencias. Algunos investigadores han observado que la supuesta correspondencia se difumina aún más respecto a la práctica docente en el aula.

También son de gran importancia algunas de las conclusiones a las que llega en dichos trabajos:

- La ausencia de reflexión previa sobre la naturaleza de la ciencia hace caer a los profesores en tópicos y contradicciones en sus ideas acerca de cómo se produce el conocimiento científico. Sus concepciones sobre este tema no están bien definidas, ni son coherentes en todos sus aspectos, por lo que no pueden considerarse inequívocamente asociadas de un modo consistente a una determinada orientación filosófica.

- $\quad$ No es posible establecer un isomorfismo general entre las concepciones de los profesores sobre la naturaleza de la ciencia y las que tienen sobre la enseñanza de las ciencias. En ocasiones podemos encontrar una correspondencia fuerte, pero en la mayoría de los casos es parcial, débil o incluso no existe.

- $\quad$ En general, no hay relación alguna entre las concepciones de los profesores sobre la naturaleza de la ciencia y su práctica docente en el aula. 
- La correspondencia entre las concepciones del profesorado acerca de la enseñanza y el aprendizaje de las ciencias con su práctica en el aula puede variar mucho, desde ninguna hasta alta, no siendo posible establecer una relación nítida entre ellas.

Estas conclusiones están en sintonía con lo señalado por Lederman (1992) en su revisión, y parece que en parte también por Porlán y Rivero (1998), cuestionando otras anteriores - y también posteriores - que muestran un paralelismo más estricto entre las concepciones del profesorado sobre la naturaleza de la ciencia, sus concepciones acerca de la enseñanza y el aprendizaje de la ciencia, y el ejercicio de la práctica docente en el aula. En particular, respecto a las creencias sobre la naturaleza de la ciencia y el desarrollo del conocimiento científico, algunas de estas discrepancias ya habían sido señaladas en otros trabajos que apuntaban hacia un panorama más complejo (Acevedo, 1994; Koulaidis y Ogborn, 1989; Lakin y Wellington, 1994). Según Koulaidis y Ogborn (1995), parte de las divergencias encontradas podrían deberse a los supuestos y las valoraciones filosóficas que los investigadores hacen en los instrumentos utilizados y también a los problemas metodológicos que se derivan de la diversidad de procedimientos empleados.

\section{ASPECTOS METODOLÓGICOS}

Se entrevistaron 24 titulados universitarios, licenciados en química (15), física (6) e ingeniería (1) y diplomados en ingeniería técnica (2), estudiantes del Curso de Actitud Pedagógica (CAP) con una experiencia docente en su mayoría prácticamente nula o, a lo sumo, pequeña. Este grupo en formación inicial constituye una muestra relativamente incidental, la de quienes participaron activamente y, además, estuvieron dispuestos a ser entrevistados fuera del horario del curso. La muestra se extrajo de un conjunto más amplio de 95 titulados universitarios, licenciados o diplomados en los estudios indicados y que también habían hecho el CAP en Huelva.

La investi gación realizada es de carácter descriptivo, interpretativo y explicativo, combinándose los métodos de análisis cualitativos con los cuantitativos (Acevedo, Acevedo, Manassero y Vázquez, 2001). En primer lugar, los profesores en formación inicial contestaron, junto a otros estudiantes del CAP, a las preguntas del Cuestionario de Opiniones sobre Ciencia y Sociedad (COCS); cuestionario de papel y lápiz y administración colectiva empleado anteriormente con estudiantes de Bachillerato Unificado Polivalente (BUP) y Curso de Orientación Universitaria (COU) (Acevedo, 1992, 1993), que consta de veinte enunciados, expresados unos en términos positivos y otros de forma negativa, relacionados con seis grandes tópicos derivados de la sociología y de la epistemología de la ciencia (puede verse su contenido completo en Acevedo, 1994).

En segundo lugar, una vez analizadas, interpretadas y clasificadas las respuestas dadas al COCS, se compararon los resultados de la muestra total (95 titulados) con los correspondientes a los que después participaron en las entrevistas (24 titulados) para comprobar la ausencia de diferencias significativas entre las respuestas de ambas muestras ( $\mathrm{A}$ y $\mathrm{B}$, respectivamente). El contraste estadístico se hizo mediante la prueba noparamétrica "chi-cuadrado" con un nivel de exigencia estadística mínimo de $p=0.05$ para poder rechazar la hipótesis de nulidad. 
En tercer lugar, se prepararon las entrevistas semi-estructurándolas en torno a preguntas problemáticas previamente decididas, relacionadas con las que se habían hecho en el cuestionario y planteadas para intentar profundizar más en el conocimiento de las creencias sobre la naturaleza de la ciencia de los futuros profesores encuestados. En principio, se estimó que las entrevistas durarían en torno a media hora cada una; pero, en la práctica, su duración real osciló entre 20 y 40 minutos aproximadamente. Todas las entrevistas se registraron en cintas de audio para su posterior transcripción, suponiendo el conjunto de las mismas casi 12 horas de grabación.

Después, en cuarto lugar, las transcripciones se trataron de acuerdo con las recomendaciones propuestas por Miles y Huberman (1984), siguiendo las pautas generales señaladas por Marcelo (1992, 1994). En este sentido cabe destacar que, para realizar el análisis de las unidades de información identificadas en las transcripciones, se establecieron previamente los códigos derivándolos directamente de las cuestiones que habían sido propuestas en la investigación, si bien se completaron en parte durante el propio proceso de análisis. Este procedimiento incluye también la reducción, clasificación y categorización de los datos de las transcripciones con el fin de darles un significado que facilitara su interpretación. Además, para disponer de una visión conjunta de los mismos y poder ultimar el análisis cualitativo, se prepararon representaciones gráficas en forma de "redes causales" (Miles y Huberman, 1984) o "mapas cognitivos" (Llinares, 1992; Mellado, 1996, 1997) de los resultados obtenidos con cada profes or en formación inicial.

Por último, se estableció una clasificación de las principales creencias de los futuros profesores a partir de las características comunes más relevantes de sus declaraciones sobre la naturaleza de la ciencia y el desarrollo del conocimiento científico, interpretadas y representadas siguiendo el proceso que acabamos de exponer sucintamente. No obstante, hay que advertir que los tipos asignados no pretenden identificar exactamente al profesorado en formación inicial en las categorías establecidas, sino sólo mostrar que los rasgos descritos recuerdan aspectos relevantes de las corrientes filosóficas y sociológicas utilizadas al establecer las categorías, sin pretender ir más allá en la analogía, como con buen criterio han señalado Mellado $(1995,1996,1997,1998)$ y, más recientemente, Petrucci y Dibar-Ure (2001).

\section{RESULTADOS OBTENIDOS}

\subsection{Respuestas al cuestionario COCS}

Por un lado (tabla 1), se indican las valoraciones derivadas de las respuestas directas dadas a las cuestiones del COCS (expresadas como frecuencias absolutas del acuerdo o desacuerdo con los enunciados propuestos) por los 24 futuros profesores de la muestra B (extraída de los 95 de la muestra A) que se entrevistaron después. 
Tabla 1

Frecuencias absolutas de las respuestas a las cuestiones del COCS y valoración grupal de la muestra B (24 profesores en formación inicial)

\begin{tabular}{|c|c|c|c|c|}
\hline Cuestiones & Acuerdo & Dudoso & Desacuerdo & Valoración grupal \\
\hline $\begin{array}{l}\text { Los modelos teóricos elaborados por } \\
\text { los científicos, por ejemplo los } \\
\text { modelos atómicos o el del ADN, } \\
\text { pretenden describir lo más exac- } \\
\text { tamente posible la realidad. }\end{array}$ & 17 & 4 & 3 & Bastante de acuerdo \\
\hline $\begin{array}{l}\text { Los mejores científicos son los que } \\
\text { siguen en sus investigaciones las } \\
\text { etapas del método científico lo más } \\
\text { escrupulosamente posible. }\end{array}$ & 12 & 7 & 5 & Algo de acuerdo \\
\hline $\begin{array}{l}\text { En general, los científicos son más } \\
\text { objetivos e imparciales en sus } \\
\text { investigaciones que la mayoría de los } \\
\text { demás ciudadanos en sus trabajos. }\end{array}$ & 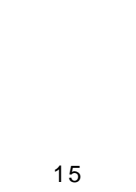 & 6 & 3 & . \\
\hline $\begin{array}{l}\text { Los contactos sociales de los } \\
\text { científicos no influyen en su trabajo } \\
\text { profesional, ni en el contenido del } \\
\text { conocimiento científico de sus } \\
\text { descubrimientos. }\end{array}$ & 5 & 8 & 11 & Algo en contra \\
\hline $\begin{array}{l}\text { La política de un país tiene poca } \\
\text { influencia sobre el trabajo de sus } \\
\text { científicos, porque sus preocupa- } \\
\text { ciones investigadoras se encuen-tran, } \\
\text { en general, al margen de la política. }\end{array}$ & 1 & 11 & 12 & En contra \\
\hline $\begin{array}{l}\text { Cuando las investigaciones cientí - } \\
\text { ficas son correctas el conocimiento } \\
\text { que se deriva de ellas no cambia } \\
\text { prácticamente en el futuro. }\end{array}$ & 2 & 9 & 13 & En contra \\
\hline
\end{tabular}


Por otro lado (tabla 2), se exponen las frecuencias absolutas y relativas (expresadas en tanto por ciento) de las creencias que, a juicio de los investigadores, se derivan de las respuestas dadas a los enunciados del Cocs tanto por la muestra A como por la B. La aplicación del contraste estadístico mediante la prueba no-paramétrica "chicuadrado" permite comprobar en todos los casos que no hay diferencias significativas entre las frecuencias de ambas muestras para el nivel establecido $(p=0.05)$. A partir de estos resultados se concluye que en el grupo de futuros profesores de la muestra B predominan los siguientes puntos de vista sobre la naturaleza de la ciencia:

- La realidad se puede describir con alguna exactitud mediante la ciencia, lo que supone asumir un cierto grado de realismo ontológico (cerca de tres de cada cuatro respuestas).

- Para acceder al conocimiento hay un potente método científico, que es jerárquicamente superior a otros desde un punto de vista epistemológico (la mitad de las respuestas).

- Los científicos tienden a ser objetivos e imparciales en su trabajo; esto es, predomina una visión objetivista de la ciencia (algo más de tres de cada cinco respuestas).

- $\quad$ El contexto sociopolítico influye en el trabajo de los científicos, en los descubrimientos que hacen y en el conocimiento que elaboran (en torno a dos de cada cinco respuestas).

- El conocimiento científico considerado correcto en un momento dado puede cambiar en el futuro (más de la mitad de las respuestas).

Tabla 2

Frecuencias absolutas y relativas (\%) de las creencias derivadas de las respuestas dadas al COCS por las muestras A (95 profesores en formación inicial) y B (24 profesores en formación inicial entrevistados)

\begin{tabular}{||l|l|l|l|l||}
\hline \multicolumn{1}{||}{ Creencias } & \multicolumn{2}{l||}{ Frec. COCS-A } & \multicolumn{2}{l||}{ Frec. COCS-B } \\
\hline $\begin{array}{l}\text { Realismo ontológico } \\
\text { Idealismo ontológico }\end{array}$ & 69 & 72.6 & 17 & 70.8 \\
10 & 10.5 & 3 & 12.5 \\
\hline Absolutismo metodológico & 41 & 43.2 & 12 & 50.0 \\
Pluralismo metodológico & 22 & 23.2 & 5 & 20.8 \\
\hline Objetivismo & 51 & 53.7 & 15 & 62.5 \\
Subjetivismo & 10 & 10.5 & 3 & 12.5 \\
\hline Contextualismo & 38 & 40.0 & 9 & 37.5 \\
Independencia del contexto & 6 & 6.3 & 2 & 8.3 \\
\hline Conocimientos cambiantes & 51 & 53.7 & 13 & 54.2 \\
Conocimientos estables & 9 & 9.5 & 2 & 8.3 \\
\hline \hline
\end{tabular}


Estas posiciones generales permiten tener una primera idea de la visión de la naturaleza de la ciencia que, como grupo, poseen estos futuros profesores antes de su formación inicial. Además, como se ha señalado más arriba, también sirven para orientar las entrevistas individuales realizadas después.

\subsection{Respuestas a las entrevistas}

En la tabla 3 se comparan las frecuencias absolutas y relativas (expresadas en tanto por ciento) de las creencias interpretadas a partir de las respuestas dadas a las preguntas del COCS por los profesores en formación inicial de la muestra B y las que se desprenden de las entrevistas hechas. Por otra parte, en el cuadro adjunto se expresan resumidos los principales rasgos y descripciones de los diferentes tipos de creencias manifestadas sobre la naturaleza de la ciencia, elaboradas a partir de las respuestas obtenidas en las entrevistas. Esta información se completa en la tabla 4 indicando la frecuencia absoluta para cada uno de los tipos clasificados, incluyendo dos casos dudosos entre los tipos C y D.

Tabla 3

Frecuencias absolutas y relativas (\%) de las creencias derivadas de las respuestas dadas al cocs y en las entrevistas por la muestra B (24 profesores en formación inicial)

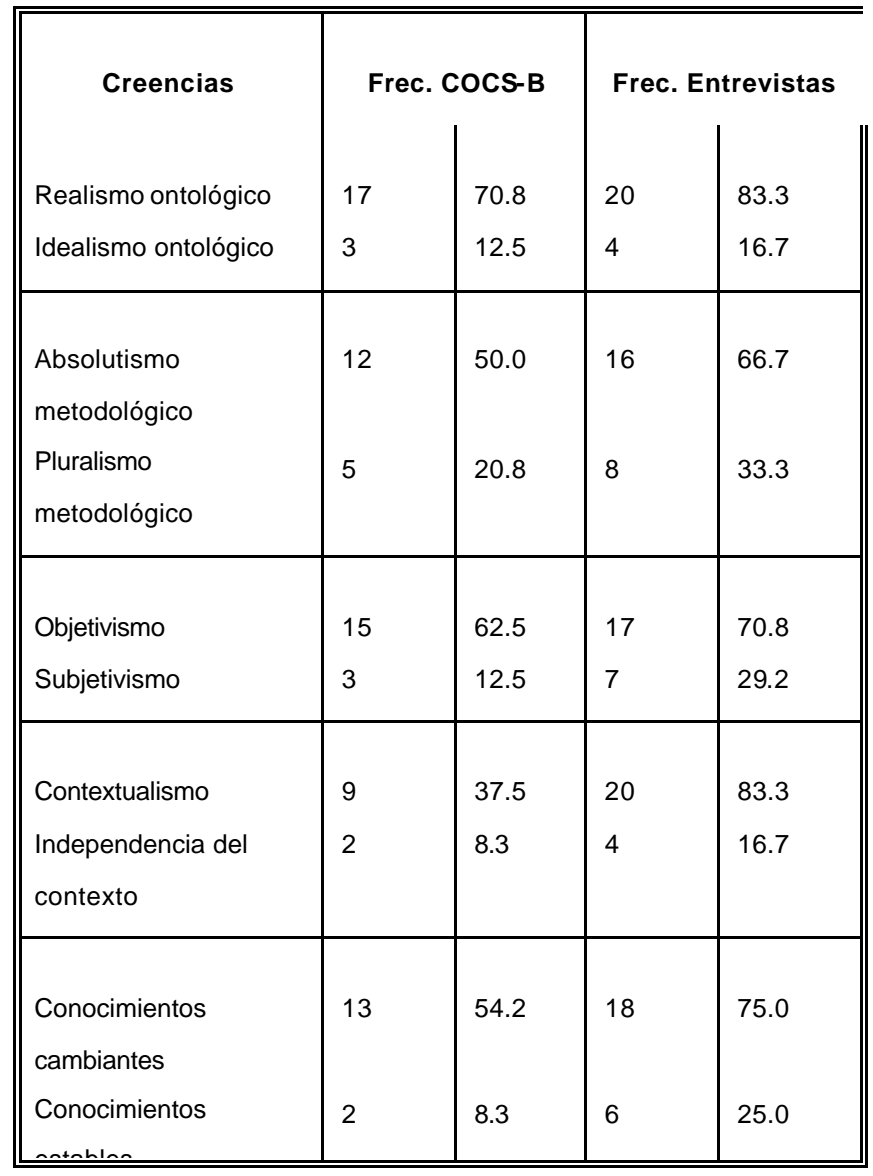




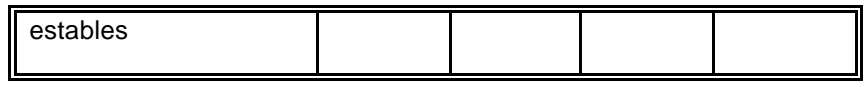

Pese a que la tipología establecida no deja de ser fruto de la interpretación de los investigadores, proporciona una descripción bastante adecuada de las creencias que los futuros profesores tienen sobre la naturaleza de la ciencia. Además, en las entrevistas se aclararon muchas de las posiciones dudosas existentes en las respuestas al COCS (alrededor de un tercio en promedio). Aunque, en general, se confirmaron algunas de las tendencias que se habían obtenido con el cuestionario (realismo ontológico, objetivismo y absolutismo metodológico), otras (perspectiva contextualista y visión dinámica del conocimiento científico) crecen aparentemente de manera notable en los resultados de las entrevistas.

\section{Cuadro}

Principales características y descripciones de la tipología elaborada a partir de las respuestas dadas en las entrevistas por la muestra B ( 24 profesores en formación inicial)

\begin{tabular}{|c|c|c|}
\hline Tipos & Rasgos & Descripciones \\
\hline A & $\begin{array}{l}\text { Idealistas ontológicos. } \\
\text { Relativistas } \\
\text { epistemológicos: } \\
\text { subjetivistas por el } \\
\text { contexto. }\end{array}$ & $\begin{array}{l}\text { La construcción del conocimiento científico depende del contexto } \\
\text { sociopolítico. La práctica científica no garantiza la objetividad de } \\
\text { la ciencia. No es posible describir una realidad única, puesto que } \\
\text { no existe; con nuestras teorías tan sólo podemos hacer } \\
\text { interpretaciones. Así, el conocimiento científico se modifica porque } \\
\text { en ocasiones cambian de manera ontológica las perspectivas } \\
\text { conceptuales con las que se interpreta el mundo. }\end{array}$ \\
\hline B & $\begin{array}{l}\text { Realistas ontológicos. } \\
\text { Relativistas } \\
\text { epistemológicos: } \\
\text { subjetivistas por el } \\
\text { contexto. }\end{array}$ & $\begin{array}{l}\text { La construcción del conocimiento científico depende del contexto } \\
\text { sociopolítico. El modo de trabajo científico no garantiza la } \\
\text { objetividad de la ciencia. Es posible hacer una descripción de la } \\
\text { realidad, pero siempre desde una determinada perspectiva. De } \\
\text { esta manera, el conocimiento científico puede cambiar aunque } \\
\text { proceda de investigaciones correctas. }\end{array}$ \\
\hline C & $\begin{array}{l}\text { Realistas ontológicos. } \\
\text { Empiristas } \\
\text { contextualistas. } \\
\text { Objetivistas y } \\
\text { positivistas. }\end{array}$ & $\begin{array}{l}\text { Aunque el contexto sociopolítico puede influir más o menos, hay } \\
\text { una realidad única que es posible describir con objetividad } \\
\text { accediendo a ella empíricamente, preferentemente por inducción, } \\
\text { mediante la utilización sistemática y rigurosa del método } \\
\text { científico. El contexto puede facilitar o dificultar esta labor; esto es, } \\
\text { algunos contextos sociales, políticos y culturales favorecen el } \\
\text { acceso al conocimiento científico, mientras que otros nos alejan de } \\
\text { él. El conocimiento científico suficientemente probado por } \\
\text { investigaciones correctas no cambia básicamente; cuando se }\end{array}$ \\
\hline
\end{tabular}




\begin{tabular}{||l|l|l||}
\hline \multirow{10}{||}{} & & $\begin{array}{l}\text { modifica no es tanto por un cambio de perspectiva en la forma de } \\
\text { ver el mundo, sino por una ampliación acumulativa del dominio } \\
\text { de aplicación de la teoría elaborada. }\end{array}$ \\
& $\begin{array}{l}\text { El contexto sociopolítico no influye en el conocimiento científico } \\
\text { correcto, porque éste es universal y se encuentra li bre de la carga } \\
\text { de subjetividad que conllevaría tal influencia. Existe una realidad } \\
\text { única que se puede describir con objetividad accediendo a ella } \\
\text { empíricamente, preferentemente por inducción, mediante la } \\
\text { utilización sistemática y rigurosa del método científico. El } \\
\text { conocimiento científico suficientemente probado por } \\
\text { investigaciones correctas no cambia básicamente; cuando se } \\
\text { Realistas ontológicos. } \\
\text { Empiristas radicales. } \\
\text { Objetivistas y } \\
\text { positivistas. }\end{array}$ & $\begin{array}{l}\text { mundo, sino por una ampliación acumulativa del dominio de } \\
\text { aplicación de la teoría elaborada. }\end{array}$ \\
\hline D
\end{tabular}

Tabla 4

Frecuencias de profesores en formación inicial (24) clasificados por sus respuestas en las entrevistas

\begin{tabular}{|c|c|}
\hline Tipos & Frecuencias \\
\hline A & 4 \\
\hline B & 4 \\
\hline C & 11 \\
\hline G & 2 \\
\hline $\mathrm{D}$ & \\
\hline D & 3 \\
\hline
\end{tabular}

Con todos estos datos se pueden resumir ahora los principales puntos de vista de los futuros profesores sobre la naturaleza de la ciencia reflejadas en las entrevistas:

- La inmensa mayoría (cinco de cada seis) se manifiesta realista desde una perspectiva ontológica o metafísica, siendo muy pocos los que expresan una visión idealista. 
- $\quad$ Predomina ampliamente (dos tercios) el absolutismo empirista, basado en la excelencia del método científico que posee un estatus jerárquicamente superior desde un punto de vista epistemológico, sobre el pluralismo metodológico.

- $\quad$ Es claramente mayoritaria (algo más de dos tercios) la visión objetivista en comparación con la subjetivista.

- Aparentemente, la gran mayoría (cinco de cada seis) muestra posiciones contextualistas, aunque este resultado se explicará más adelante.

- Una importante mayoría (tres de cada cuatro) tiene un punto de vista dinámico del conocimiento científico, si bien este resultado también se aclarará después.

Los resultados obtenidos en las entrevistas son, pues, más ricos y finos que los que proporciona la aplicación del cuestionario y permiten matizar algunos de los aspectos más discutibles, tal y como se refleja en la tabla 5.

Tabla 5

Frecuencias absolutas y relativas (\%) de matices de las principales creencias derivadas de las respuestas dadas en las entrevistas por los profesores en formación inicial de la muestra $B$

(24)

\begin{tabular}{|c|c|c|c|c|}
\hline Dimensiones & \multicolumn{2}{|c|}{ Creencias } & \multicolumn{2}{|c|}{ Frecuencias } \\
\hline \multirow{3}{*}{$\begin{array}{l}\text { Posiciones acerca de la } \\
\text { excelencia del método } \\
\text { científico }\end{array}$} & \multirow[b]{2}{*}{$\begin{array}{l}\text { Pluralismo } \\
\text { metodológico }\end{array}$} & Subjetivista & 7 & 29.2 \\
\hline & & Objetivista & 1 & 4.1 \\
\hline & $\begin{array}{l}\text { Absolutismo } \\
\text { metodológico }\end{array}$ & Objetivista & 16 & 66.7 \\
\hline \multirow{3}{*}{$\begin{array}{l}\text { Puntos de vista sobre la } \\
\text { influencia del contexto }\end{array}$} & \multicolumn{2}{|c|}{$\begin{array}{l}\text { Contextualismo internalista y } \\
\text { externalista }\end{array}$} & 8 & 33.3 \\
\hline & \multicolumn{2}{|c|}{ Contextualismo externalista } & 12 & 50.0 \\
\hline & \multicolumn{2}{|c|}{ Independencia del contexto } & 4 & 16.7 \\
\hline $\begin{array}{l}\text { Visión del cambio del } \\
\text { conocimiento científico }\end{array}$ & \multicolumn{2}{|c|}{$\begin{array}{l}\text { Cambio conceptual ontológico y } \\
\text { metodológico }\end{array}$} & 8 & 33.3 \\
\hline
\end{tabular}




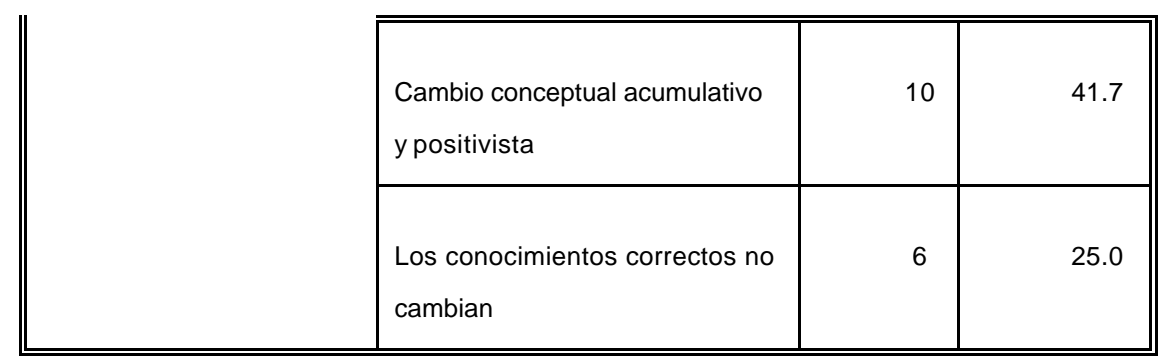

\section{DISCUSIÓN DE LOS RESULTADOS}

Desde un punto de vista epistemológico, aproximadamente un te rcio de los futuros profesores de ciencias se muestra subjetivista por el contexto; esto es, sus declaraciones se ajustan más al relativismo epistemológico característico del constructivismo sociológico postmoderno, puesto que se tiende más a hacer depender la validez del conocimiento no tanto de factores internos al sujeto (subjetivismo psicológico; por ejemplo,, el del idealismo subjetivo de Berkeley) como de los externos (factores contextuales; por ejemplo,, los aspectos históricos, sociales, políticos, culturales y económicos). Sin embargo, a la vez, la mayor parte del profesorado en formación inicial se muestra realista desde un punto de vista ontológico, lo que no debe sorprendernos demasiado porque incluso la mayoría de los defensores del constructivismo en la enseñanza y el aprendizaje de las ciencias, aunque sea subjetivista desde una perspectiva epistemológica (más propiamente relativista, de acuerdo con lo que acabamos de expresar), es realista ontológico; esto es, considera que la realidad existe al margen de lo que se piense sobre ella, aunque no se pueda conocer con objetividad. Esta posición puede verse claramente expuesta, por ejemplo, en Driver y Oldham (1986):

[...] La visión de la ciencia actualmente dominante asegura que, aunque pueda asumirse la realidad de un mundo externo, no tenemos acceso directo a él; la ciencia como conocimiento de dominio público no es tanto un descubrimiento como una construcción cuidadosamente comprobada. (p. 109).

Y de algún modo también en Porlán (1995, reproducido así mismo en Porlán y Martín del Pozo, 1996; p. 26):

[...] Una imagen relativizadora de la ciencia, según la cual el conocimiento científico se construye por procesos de creación y comprobación de teorías que cambian y evolucionan históricamente y que sólo son aproximaciones tentativas y parciales sobre determinados aspectos de la realidad." (p. 12).

No obstante, algunos partidarios del constructivismo filosófico radical, como Von Glasersfeld (1987), no solamente defienden el relativismo epistemológico sino una cierta clase de idealismo ontológico. Según Matthews (1992, 1994b), los argumentos esenciales de esta forma de constructivismo radical no son más que una versión actualizada de los sostenidos por Berkeley en su idealismo ontológico, psicológico (subjetivo) y en buena parte empírico. Desde luego, la posición filosófica de pensadores como Berkeley y Vico, que son referentes destacados por Von Glasersfeld $(1987,1995)$ y sus seguidores, casa muy mal con los planteamientos de la ciencia moderna de Galileo y Newton, a los que se oponían con rotundidad (Matthews, 1992, 1994b; Suchting, 1992), tal y como se desprende del revelador subtítulo del Tratado relativo a los principios del conocimiento humano de Berkeley 
(publicado en 1710): "En donde se indagan las principales causas del error y las dificultades de las ciencias, basadas en el escepticismo, el ateísmo y la irreligión".

Por otro lado, la mayoría de los futuros profesores (en torno a dos de cada tres) cree en la objetividad de la ciencia y de los científicos en su trabajo, asociando su objetivismo a la existencia de un potente, riguroso y universal método científico (tabla 5), que permite acceder al conocimiento de manera empírica y preferentemente inductiva, de acuerdo con la clásica secuencia Observación-Hipótesis-Experimentación-Teoría (O-HE-T). Así pues, para estos profesores en formación inicial, es más bien la metodología característica de las ciencias experimentales la que las hace más objetivas para elaborar un conocimiento válido y fiable, radicando su eficacia en la posibilidad de someter los descubrimientos al control de la comunidad científica mediante la réplica imparcial de las investigaciones. Por lo tanto, respecto a esta cuestión, puede decirse sin ambigüedades que la mayoría manifiesta una posición epistemológica bastante coherente, acorde con uno de los constructos sobre el pensamiento del profesor de ciencias identificado, entre otros, por Gallagher (1991) en el profesorado en ejercicio de educación secundaria y por Abell y Smith (1994) y Porlán (1994; Porlán y Martín del Pozo, 1996; Porlán y Rivero, 1998) en los estudiantes de profesorado de educación primaria.

En este estudio, la mayor parte de los futuros profesores entrevistados (dos de cada tres), además del objetivismo, tiene puntos de vista epistemológicos próximos al empirismo y al positivismo, aunque son pocos los que se manifiestan empiristas radicales si se comparan con los que admiten una cierta clase de contextualismo, pero con un sentido bastante diferente al de los relativistas epistemológicos (cuadro y tablas 4 y 5). En relación con esto, Koulaidis y Ogborn (1989) señalaron que los profesores de ciencias suelen asumir posiciones eclécticas sobre la naturaleza de la ciencia, más próximas al contextualismo de Kuhn que al empirismo. Este resultado contrasta con los de otras investigaciones como, por ejemplo, la de Ruggieri, Tarsitani y Vicentini (1993), quienes, trabajando con profesores de ciencias y matemáticas (en su mayoría físicos) italianos, argentinos y uruguayos, encontraron menos sensibilidad, sobre todo en los italianos, a la influencia de los factores de contexto (sociales, culturales y económicos) en la elaboración y evolución del conocimiento científico, y también a los aspectos sociológicos ligados a su cons trucción (como puede ser la importancia del consenso científico). En nuestro trabajo, al igual que ocurre en otros recientes desarrollados en España (por ejemplo,, Rebollo, 1998), también se encuentra un elevado porcentaje de referencias al contexto en los futuros profesores entrevistados. Sin embargo, no todas éstas pueden considerarse contextualistas en el sentido que le dan al término Kuhn (1962) o la posterior sociología constructivista del conocimiento científico.

En efecto, la mayor parte (tres de cada cinco) de los que admiten la influencia de aspectos, sociales, políticos, etc., lo hacen asignándole un significado diferente (cuadro y tablas 4 y 5). Consideran que en algunos contextos socioculturales, como el de la civilización occidental, se apoya p úblicamente la ciencia creando el marco adecuado, aportando fondos y subvenciones, y dando reconocimiento a los científicos más destacados, premiándolos con honores y recompensas. De esta manera se protege y promociona, en general, el conocimiento científico. Ahora bien, para estos profesores en formación inicial, una vez establecido este contexto externo ampliamente favorable a las ciencias experimentales, el conocimiento que éstas elaboran pertenece a otra esfera interna en la que imperan el empirismo, la universalidad y la objetividad individual y, más aún, institucional como 
características esenciales del método científico, que adquiere así un estatus jerárquicamente superior a otras formas de acceder al conocimiento. Esta excelencia del método científico ha sido puesta de manifiesto en muchos estudios sobre las creencias del profesorado acerca de la naturaleza de la ciencia y el desarrollo del conocimiento científico (Rebollo, 1998; Ruggieri, Tarsitani y Vicentini, 1993; Thomaz, Cruz, Martins y Cachapuz, 1996). Por otra parte, los demás profesores en formación inicial (un tercio del total, equivalente a dos de cada cinco de los que se manifiestan contextualistas en apariencia) sí lo hacen desde una perspectiva epistemológica relativista; es decir, opinan que también el propio conocimiento científico se ve intrínsecamente afectado por los factores culturales, sociales, históricos y políticos (cuadro y tablas 4 y 5 ).

Otra cuestión importante abordada en este trabajo es la visión del cambio del conocimiento científico que tienen los futuros profesores. En relación con esto, Rebollo (1998) ha proporcionado resultados -obtenidos mediante un cuestionario - que muestran que la práctica totalidad de las personas de su investigación, licenciados en Biología o en Quí mica y estudiantes del CAP de la Universidad de Málaga, admite el cambio de conceptos y teorías científicas, concediendo un estatus temporal al conocimiento científico. Esta visión dinámica del conocimiento científico es asumida también por una gran mayoría de estudiantes portugueses de profesorado de educación primaria (Thomaz, Cruz, Martins y Cachapuz, 1996). Por el contrario, aunque en principio más de la mitad de los futuros profesores encuestados con el COCS está bastante o totalmente de acuerdo con que el conocimiento procedente de investigaciones científicas correctas puede cambiar en el futuro y solamente una minoría muy pequeña se muestra en desacuerdo (tabla 2), después de las entrevistas se pone claramente de manifiesto que, si bien en apariencia aumentaba el número de los primeros (tabla 3), tan sólo uno de cada tres sostiene que dos teorías puedan ser formal y sustancialmente correctas porque se aproximan al mundo real desde perspectivas globales distintas (cuadro y tablas 4 y 5 ); esto es, porque tales teorías constituyen dos paradigmas diferentes en el sentido de Kuhn (1962). Un ejemplo de este tipo es el de las teorías gravitatorias de Newton y Einstein, otro es el de la teoría electromagnética de la acción a distancia de Coulomb y Ampère frente a la del campo electromagnético de Faraday y Maxwell; en ambos casos, hay un cambio metafísico u ontológico en la forma de "ver la realidad".

Como puede observarse en la tabla 5 , los restantes profesores en formación inicial que están de acuerdo en que el conocimiento científico aun siendo correcto puede cambiar con el tiempo, que son mayoría relativa (en torno a dos de cada cinco), lo hacen más bien desde una perspectiva positivista y acumulativa del conocimiento, que es otro de los rasgos señalado por Porlán (1994). En efecto, en las entrevistas solventan esta cuestión problemática justificando que dos teorías que se ocupan de un mismo fenómeno pueden ser válidas al mismo tiempo bien porque abordan parcelas distintas de la realidad y son complementarias, bien porque una de ellas termina por quedar reducida a un caso particular de la otra, de acuerdo con el principio de simplicidad y máxima economía del conocimiento, que es una forma de "reduccionismo epistemológico".

A pesar del carácter relativamente incidental de la muestra de este estudio, lo que no permite una generalización de los resultados indiscriminada, éstos son suficientemente válidos y fiables, dentro del marco y los criterios metodológicos utilizados, para identificar tendencias más nítidas s obre la naturaleza de la ciencia en las creencias de los titulados universitarios (licenciados y diplomados) que están en formación inicial para ser profesores de ciencias de educación secundaria. Se han comparado estos resultados con los de otros trabajos, 
sobre todo del contexto cultural español y otros más o menos próximos (por ejemplo, Portugal, Italia, Argentina y Uruguay), que utilizan fundamentalmente cuestionarios, preguntas abiertas y, a veces, también entrevistas, comprobando en ocasiones la concordancia con los mismos y dando algunas explicaciones tentativas a las posibles discordancias encontradas, incluyendo también las que hay en nuestro propio estudio respecto a la aplicación del cocs.

\section{CONCLUSIONES, IMPLICACIONES EDUCATIVAS Y PERSPECTIVAS FUTURAS DE INVESTIGACIÓN}

A la vista de los resultados obtenidos, se pueden establecer dos grupos generales de conclusiones más importantes, cada uno con sus propias implicaciones y perspectivas de futuro.

\subsection{Sobre la metodología}

1.1 La técnica de entrevistas grabadas en cintas de audio ha sido bastante adecuada para los propósitos pretendidos, puesto que permite alcanzar un conocimiento mucho más fino y matizado del tema que el proporcionado por el cuestionario cocs.

1.2 Sin embargo, debido a que el número de participantes en el estudio es relativamente elevado, el esfuerzo realizado y el tiempo empleado en grabaciones, transcripciones y su posterior tratamiento metodológico es demasiado grande respecto al uso del cuestionario.

1.3 La utilización del COCS resulta válida como primera aproximación y para orientar las preguntas de las entrevistas; su potencial se incrementa cuanto mayor es el tamaño de la muestra.

1.4 Por otra parte, el COCS también puede ser bastante útil para iniciar debates sobre las cuestiones CTS que se abordan en los cursos de formación del profesorado.

Al igual que ha ocurrido en otras ramas de la investigación educativa, en general, y de la didáctica de las ciencias, en particular, con el paso de los años la investigación sobre las creencias acerca de la naturaleza de la ciencia se ha ido desplazando lentamente desde los enfoques cuantitativos a otros más cualitativos (Lederman, 1992). Los primeros trabajos utilizaban sobre todo escalas de Likert, diferenciales semánticos y cuestionarios de opción múltiple, generalmente diseñados desde la óptica del investigador. A partir de la segunda mitad de los años ochenta estos instrumentos fueron muy criticados por variados motivos (Manassero, Vázquez y Acevedo, 2001a,b). Por un lado, los resultados que proporcionaban dejaban un amplio margen de inseguridad; por otro, se asumía erróneamente que, al leer los enunciados de las cuestiones, las personas encuestadas entendían lo mismo que el investigador que había diseñado el instrumento — lo que se ha denominado con ironía la inmaculada percepción-. Así pues, no es de extrañar que se produjera un giro metodológico hacia el paradigma de la investigación cualitativa, ganando adeptos las entrevistas clínicas porque en general son muy adecuadas para la investigación en educación y suelen dar una información bastante segura en comparación con otros instrumentos de papel y lápiz (Aikenhead, 1988). La proliferación del uso de técnicas cualitativas permitió a los investigadores, entre otras cosas, evitar los problemas creados por las limitaciones de las respuestas a los instrumentos cuantitativos a la hora de encajarlas en categorías o puntos de vista establecidos a priori.

OEI-Revista Iberoamericana de Educación (ISSN: 1681-5653) 
No obstante, pese a estas críticas, cada vez hay un consenso mayor para combinar las inv estigaciones cualitativas con las cuantitativas (Acevedo, Acevedo, Manassero y Vázquez, 2001). Como hemos señalado más arriba, las entrevistas clínicas también tienen sus limitaciones; entre otras, son muy costosas en tiempo y esfuerzo, por lo que su aplicación tiene que restringirse necesariamente a un número pequeño de personas. No es de extrañar, por tanto, que haya continuado la elaboración de cuestionarios aplicables a grandes muestras, pero construyéndose ahora empíricamente siguiendo las pautas del paradigma de la investigación cualitativa para no volver a caer en errores anteriores; especialmente, el peligro potencial de que las personas investigadas no perciban el mismo significado de las preguntas que el investigador. En efecto, los items de opciones múltiples desarrolladas empíricamente pretenden averiguar los puntos de vista de las personas que tienen que responder al cuestionario tratando de no influir con la perspectiva del investigador. Su principal característica es que las opciones múltiples recogen las opiniones expresadas en entrevistas piloto anteriores o las repuestas abiertas escritas por personas semejantes a las que está destinado el cuestionario.

El instrumento de esta clase más conocido, que incluye diversas cuestiones sobre la naturaleza de laciencia, es el "Views on Science-Technology-Society" (VOSTS), preparado por Aikenhead, Fleming y Ryan (1987) y modificado poco después (Aikenhead y Ryan, 1992; Aikenhead, Ryan y Fleming; 1989). Los motivos que llevaron a estos investigadores canadienses a elaborar empíricamente este cuestionario de opción múltiple y valoración con respuesta única, los pasos que siguieron para desarrollarlo y una discusión sobre su validez y fiabilidad se tratan en los artículos citados y en otros de los mismos autores (por ejemplo,, Aikenhead, 1988, Aikenhead y Ryan, 1989). Partiendo de una taxonomía de actitudes relacionadas con la ciencia y la tecnología propuesta por Vázquez y Manassero (1995b), se ha preparado una adaptación del VOSTS en español: el Cuestionario de Opiniones sobre Ciencia, Tecnología y Sociedad (COCTS), con veinte cuestiones sobre la naturaleza de la ciencia entre las cien correspondientes a diferentes aspectos CTS (Manassero, Vázquez y Acevedo, 2001a; Vázquez y Manassero, 1997).

Desde hace p oco tiempo los esfuerzos se están dirigiendo a desarrollar nuevos modelos para valorar las respuestas de opción múltiple de estos cuestionarios. Una vez que establecieron tres categorías para clasificar las opciones múltiples, Rubba y Harkness (1993) ensay aron la aplicación de métodos tradicionales del análisis de datos a items semejantes a los del VOSTS. Poco después, Rubba, Schoneweg-Bradford y Harkness (1996) propusieron una nueva forma de puntuar las opciones del VOSTS, que fue cuestionada y mejorada posteriormente por Vázquez y Manassero (1999a,b). Estos autores también plantean perfeccionar la evaluación utilizando un "modelo de respuesta múltiple", que valora todas y cada una de las opciones de las cuestiones, frente a los "modelos de respuesta única" que han venido utilizando los investigadores canadienses y estadounidenses citados (Acevedo, Manassero y Vázquez, 2001). Estos nuevos procedimientos se enmarcan en la tradición de la psicología social para la evaluación de actitudes y creencias conocida c omo "escalamiento psicofísico", una alternativa a las técnicas de evaluación psicométricas que se siguen, por ejemplo, cuando se aplican escalas de Likert o diferenciales semánticos (Manassero, Vázquez y Acevedo, 2001a; Vázquez, Acevedo y Manassero, 2000).

Es muy necesario, pues, continuar progresando en la elaboración de instrumentos adecuados y avanzar en las técnicas para evaluar las creencias sobre la naturaleza de la ciencia. El uso de cuestionarios empíricamente 
desarrollados (como, por ejemplo,, el VOSTS y el COCTS), que permiten al mismo tiempo valorar los diversos items de acuerdo con un modelo de respuesta múltiple, podría servir quizás para aunar las ventajas de las tradiciones de investigación cualitativa y cuantitativa, atemperando al mismo tie mpo las desventajas propias de cada una de ellas. Evaluar las creencias acerca de la naturaleza de la ciencia con instrumentos y procedimientos de este tipo, que tratan de evitar la influencia de las percepciones del investigador y dan la máxima informació n posible sobre cada cuestión, permitirá, sin duda, seguir avanzando en este importante campo de investigación de la didáctica de las ciencias experimentales.

\subsection{Sobre las creencias}

2.1 Predominan algunas creencias ya mostradas en otros trabajos, tales como realismo, objetivismo, estatus jerárquicamente superior del método científico, empirismo, visión acumulativa del conocimiento científico, positivismo, etc.

2.2 Pese al intento de agrupar al profesorado en formación inicial en unos pocos tipos de rasgos, los resultados revelan un panorama más rico y complejo que el aportado por otras investigaciones sobre el tema, no sólo por la presencia minoritaria, pero significativa, de algunos puntos de vista epistemológicos contrarios a los anteriores: pluralismo metodológico, subjetivismo, relativismo, etc., sino por ciertos matices que aclaran diferentes posiciones en relación con la visión cambiante del conocimiento científico y la posible influencia de factores sociales, culturales y políticos en la ciencia y lo s conocimientos que ésta elabora.

Se critica a menudo al profesorado y, en menor medida quizás, a los estudiantes por sus concepciones inadecuadas sobre la naturaleza de la ciencia; por ejemplo, ambos colectivos han sido censurados por no comprender correctamente la provisionalidad del conocimiento científico. Ahora bien, si se tienen en cuenta las diferencias que hay entre las posiciones epistemológicas de filósofos como Popper, Kuhn, Lakatos, Feyerabend, Toulmin, Laudan, Putnan, Van Fraassen, Chalmers, Hacking, etc. (Vázquez, Acevedo, Manassero y Acevedo, 2001), englobados con frecuencia dentro de la que ha sido denominada como Nueva Filosofía de la Ciencia (Brown, 1977), se pone claramente de manifiesto que no hay una sola forma de concebir la naturaleza de laciencia y que ésta es tan provisional, al menos, como el propio conocimiento científico (Vázquez, Acevedo y Manassero, 2001). Los puntos de vista sobre ésta han ido cambiando a lo largo del tiempo y probablemente continuarán haciéndolo en el futuro, sobre todo en lo que concierne a cómo se desarrolla el conocimiento científico.

Algunas investigaciones recientes sobre las creencias acerca de la naturaleza de la ciencia, como la desarrollada en este artículo, muestran que el profesorado (en ejercicio o en formación inicial) presenta, en conjunto, una amplia variedad de puntos de vista sobre el tema, con diversos grados de complejidad y coherencia también variable. Del mismo modo, los propios filósofos y sociólogos de la ciencia también manifiestan impo rtantes desacuerdos a la hora de conceptualizar los principios básicos de la naturaleza de la ciencia (Alters, 1997; Eflin, Glennan y Reisch, 1999), posiblemente como consecuencia de su propio carácter dialéctico y controvertido (Vázquez, Acevedo, Manasser o y Acevedo, 2001). Como ha subrayado Matthews (1998), desde el nacimiento de la ciencia moderna los debates sobre la forma de concebir su naturaleza se han producido siempre y continúan 
vivos en la actualidad ${ }^{1}$ debido a la diversidad de puntos de vista existentes (como casos paradigmáticos de una visión contraria a la emanada de la epistemología y la sociología postmodernas constructivistas véanse, por ejemplo,, Bunge, 1999; Sokal y Bricmont, 1998; Wolpert, 1992).

Nuestra posición es opuesta al adoctrinamiento de quienes pretenden imponer una determinada perspectiva de la naturaleza de la ciencia a profesores y estudiantes, presentándola como si fuera la mejor o inmutable. Por el contrario, aquí se defiende la necesidad de mostrar al profesorado y alumnado diversos puntos de vista sobre el tema, dándoles a conocer las distintas formas de entender la naturaleza de la ciencia para que puedan comprenderla mejor, valorarla críticamente $y$, sobre todo, adquirir la idea clave de que las conceptualizaciones sobre la naturaleza de la ciencia también cambian, tal y como ocurre con los propios conceptos científicos (Vázquez y Manassero, 1995a). Todo esto supone descartar los enfoques formativos reduccionistas, sesgados hacia el estudio de una sola corriente de pensamiento como sumo paradigma capaz de explicar los planteamientos sociales o filosóficos de la ciencia; por el contrario, es necesario presentar al profesorado una variedad de autores, pensamientos, opiniones y enfoques para que puedan someterlos a un reflexivo análisis crítico (Vázquez, Acevedo, Manassero y Acevedo, 2001). Se cumpliría así el objetivo de que la introducción en la formación del profesorado de la reflexión epistemológica sobre la ciencia conduzca a que se adquiera una visión más plural, evitando en lo posible posturas más o menos dogmáticas (Jiménez, 1995).

Como ha señalado Rebollo (1998), la importancia de que los profesores de ciencias reflexionen sobre la naturaleza de la ciencia no suele asumirse durante su formación universitaria, lo que en muchos casos puede llegar a suponer la posterior falta de interés y hasta el rechazo de los contenidos relacionados con esta temática, tal y como ha informado De Pro (1995) en un estudio hecho con profesores tutores de prácticas del CAP de la Universidad de Murcia. En otros trabajos se ha resaltado que, puesto que la formación inicial del profesorado en estas cuestiones suele ser generalmente escasa y a menudo también obsoleta, es urgente su incorporación explícita a los planes de formación inicial y de actualización científica y didáctica para la formación permanente del profesorado, con el fin de tratar de promover la reflexión del profesorado sobre estos aspectos y favorecer su adaptación curricular (Acevedo, 1994, 1996). En efecto, aunque algunos autores sostienen la formación implícita (Palmquist y Finley, 1997, 1998) y otros han mostrado recientemente la influencia positiva de los mensajes implícitos en el aprendizaje de la naturaleza de la ciencia (Moss, Abrams y Robb, 2001; Ryder y Leach, 1999), hay un consenso bastante generalizado en que una formación exclusivamente implícita no permite una buena comprensión de la naturaleza de la ciencia (AAAS, 1993; Abd-El-Khalick, Bell y Lederman, 1998; Abd-El Khalick y Lederman, 2000a, 2000b; Akerson, Abd-El-Khalick y Lederman, 2000; Bell, Lederman y Abd-EF-Khalick, 1998, 2000; Lederman, 1992, 1999; Lederman y Abd-El-Khalick, 1998; Monk y Osborne, 1997).

Esta necesaria reflexión epistemológica sobre la ciencia y el conocimiento científico, que implica conocer al menos algunas de las principales aportaciones provenientes de las perspectivas filosófica, histórica y sociológica,

${ }^{1}$ Pueden verse ejemplos de interés para la enseñanza de las ciencias en la revista Science \& Education que, además de publicar con frecuencia artículos sobre el tema en sus diez volúmenes, le ha dedicado dos números monográficos: el 6(4) en 1997 y el 7(6) en 1998, respectivamente editados por M.R. Matthews y N.G. Lederman, W.F. McComas y M.R. Matthews 
podría contribuir a mejorar la fundamentación didáctica de las materias científicas (Vázquez, Acevedo y Manassero, 2001; Vázquez, Acevedo, Ma nassero y Acevedo, 2001). Pero, desde la didáctica de las ciencias, resulta imprescindible tratar estos temas relacionándolos con la propia práctica docente (Mellado y Carracedo, 1993); esto es, abordarlos en el marco de la enseñanza y el aprendizaje de la s ciencias, porque la inmensa mayoría de las cuestiones relacionadas con la docencia no adquieren plenamente su sentido hasta que el profesor no se enfrenta con ellas en la compleja realidad del aula (Acevedo, 1996). En cualquier caso, para apoyar o cuestionar las propuestas didácticas de los cursos de formación del profesorado de ciencias, es de particular interés que los futuros profesores manifiesten y aborden de manera explícita cómo conciben la naturaleza de la ciencia y la elaboración del conocimiento científico (García Estañ, De Pro y Valcárcel, 1999).

No obstante, aunque la posesión por parte del profesorado de una visión filosófica y sociológicamente más rica y reflexiva sobre la ciencia y el trabajo científico sea muy necesaria, no conviene perder de vista que esto no garantiza que sus estudiantes vayan a lograr una mejor comprensión de la naturaleza de la ciencia y del desarrollo del conocimiento científico, de modo similar al conocido hecho de que el conocimiento de los profesores de la materia a enseñar, aunque es muy importante, tampoco asegura en absoluto un buen aprendizaje de la misma por parte de los alumnos (Acevedo, 1996). En todo esto inciden otros muchos y complejos factores que, sin duda, hacen perder gran parte de la coherencia del discurso epistemológico cuando se pasa del plano teórico (lo que se dice que hay que hacer) al desarrollo de la práctica en el aula (lo que realmente se hace).

Para terminar, señalaremos que los estudios de las creencias del profesorado (ya sea en formación inicialo en ejercicio) sobre la naturaleza de la ciencia deben ampliarse incluyendo también sus puntos de vista acerca de la naturaleza de la tecnología y del desarrollo del conocimiento tecnológico, porque en los albores del siglo XXI la tecnología, con estatus, características y racionalidad propias, está influyendo cada vez más en la ciencia, tanto en el modo de entenderla como en el de hacerla (Acevedo, 1997). Si bien todavía es posible utilizar algunos criterios para distinguir entre ciencia y tecnología (Acevedo, 1998a,b), no es menos cierto que las fronteras entre ambas están difuminándose rápidamente; fenómeno que suele conocerse como tecnociencia. A nuestro juicio, todo esto originará, si no lo ha hecho ya, nuevas formas de conceptualizar la naturaleza de la ciencia, de acuerdo con la idea de su carácter cambiante. Se abren así, nuevas perspectivas para esta línea de investigación per se(Vázquez, Manassero y Acevedo, 1998), las cuales adquieren indudablemente todo su sentido dentro del marco teórico de la educación CTS.

\section{REFERENCIAS BIBLIOGRÁFICAS}

AAAS (1990): Science for all americans. A project 2061 report on literacy goals in Science, Mathematics, and Technology. Nueva York, Oxford University Press.

AAAS (1993): Benchmarck for science literac y. A project 2061 report. Nueva York, Oxford University Press.

ABD-EL-KHALICK, F.; BELL, R.L., y LEDERMAN, N.G. (1998): "The nature of science and instructional practice: making the unnatural natural", en: Science Education, 82(4), 417-436.

OEI-Revista Iberoamericana de Educación (ISSN: 1681-5653) 
ABD-EL-KHALICK, F., y LEDERMAN, N. G., (2000a): "Improving science teachers' conceptions of nature of science: a critical review of the literature", en: International Journal of Science Education, 22(7), 665-701.

ABD-EL-KHALICK, F., y LEDERMAN, N.G. (2000b): "The influence of History of Science course on students' views of nature of science", en: Journal of Research in Science Teaching, 37(10), 1057-1095.

ABELL, S.K., y SMITH, D.C. (1994): "What is science? Preservice elementary teachers' conceptions of the nature of science", en: International Journal of Science Education, 16(4), 475-487.

ACEVEDO, J.A. (1992): "Cuestiones de sociología y epistemología de la ciencia. La opinión de los estudiantes", en: Revista de Educación de la Universidad de Granada, 6, 167-182.

ACEVEDO, J.A. (1993): “¿Qué piensan los estudiantes sobre la ciencia? Un enfoque CTS”, en: Enseñanza de las Ciencias, n.ำ extra (IV Congreso), 11-12.

ACEVEDO, J.A. (1994): "Los futuros profesores de enseñanza secundaria ante la sociología y la epistemología de las ciencias", en: Revista Interuniversitaria de Formación del Profesorado, 19, 111-125. Versión electrónica corregida y actualizada en Sala de Lecturas CTS+l de la OEI <http://www.campusoei.org/salactsi/acevedo8.htm>, 2001.

ACEVEDO, J.A. (1996): "La formación del profesorado de enseñanza secundaria y la educación CTS. Una cuestión problemática", en: Revista Interuniversitaria de Formación del Profesorado, 26, 131-144. Versión electrónica corregida y actualizada en Sala de Lecturas CTS+l de la OEI <http://www.campusoei.org/salactsi/acevedo9.htm>, 2001.

ACEVEDO, J.A. (1997): “¿Publicar o patentar? Hacia una ciencia cada vez más ligada a la tecnología”, en: Revista Española de Física, 11(2), 8-11. Versión electrónica corregida y actualizada en Sala de Lecturas CTS+lde la OEI <http://www.campus-oei.org/salactsi/acevedo4.htm>, 2001.

ACEVEDO, J.A. (1998a): "Análisis de algunos criterios para diferenciar entre ciencia y tecnología", en: Enseñanza de las ciencias, 16(3), 409-420.

ACEVEDO, J.A. (1998b): "Tres criterios para diferenciar entre ciencia y tecnología”, en E. Banet, y A. de Pro (eds.): Investigación e innovación en la enseñanza de las ciencias. Vol I. DM Murcia, 7-16.

ACEVEDO, J.A.; ACEVEDO, P.; MANASSERO, M.A., y VÁZQUEZ, A. (2001): "Avances metodológicos en la investigación sobre evaluación de actitudes y creencias CTS", en: Revista Iberoamericana de Educación, edición electrónica De los Lectores (4-6-2001) <http://www.campus-oei.org/revista/deloslectores/Acevedo.PDF>.

ACEVEDO, J.A.; VÁZQUEZ, A.; MANASSERO, M.A., y ACEVEDO, P. (2002): Persistencia de las actitudes y creencias CTS en la profesión docente. Enviado para su publicación. 
AIKENHEAD, G.S. (1988): "An analysis of four ways of assessing student beliefs about STS topics", en: Journal of Research in Science Teaching, 25(8), 607-629.

AIKENHEAD, G.S. (1997): "Student views on the influence of culture on science", en: International Journal of Science Education, 19(4), 419-428.

AIKENHEAD, G.S.; FLEMING, R.W., y RYAN, A.G. (1987): "High -school graduates' beliefs about science technology -society. I. Methods and issues in monitoring student views", en: Science Education, 71(2), 145-161.

AIKENHEAD, G.S., y RYAN, A.G. (1989): The development of a multiple choice instrument for monitoring views on Science-Technology-Society topics. Saskatoon (Canadá), Department of Curriculum Studies, College of Education, University of Saskatchewan.

AIKENHEAD, G.S., y RYAN, A.G. (1992): The development of a new instrument: "views on ScienceTechnology-Society" (VOSTS), en: Science Education, 76(5), 477-491.

AIKENHEAD, G.S.; RYAN, A.G., y FLEMING, R.W. (1989): Views on Science-Technology-Society (form CDN. mc. 5). Saskatoon (Canadá), Department of Curriculum Studies, College of Education, University of Saskatchewan.

AKERSON, V.L.; ABD-EL-KHALICK, F., y LEDERMAN, N.G. (2000): "Influence of a reflective explicit activitybased approach on elementary teachers' conceptions of nature of science", en: Journal of Research in Science Teaching, 37(4), 295-317.

ALTER S, B.J. (1997): “Whose nature of science?”, en: Journal of Research in Science Teaching, 34(1), 3955.

BELL, R.L.; LEDERMAN, N. G., y ABD-EL-KHALICK F. (1998): "Implicit versus explicit nature of science instruction: an explicit response to Palmquist and Finley", en: Journal of Research in Science Teaching, 35(9), 1057-1061.

BELL, R.L.; LEDERMAN, N.G., y ABD-EL-KHALICK F. (2000): "Developing and acting upon one's conception of the nature of science: a follow -up study", en: Journal of Research in Science Teaching, 37(6), 563-581.

BRICKHOUSE, N.W. (1990): "Teacher' beliefs about the nature of science and their relationship to classroom practice", en: Journal of Teacher Education, 41(3), 53-62.

BROWN, H.I. (1977): Perception, theory and commitment. The new philosophy of science. Chicago, University of Chicago Press; traducción de J.M. Domínguez y J. Sánchez Cuenca (1983): La nueva filosofía de la ciencia. Madrid, Tecnos.

OEI-Revista Iberoamericana de Educación (ISSN: 1681-5653) 
BUNGE, M. (1999): “The sociology-philosophy connection. New Brunswick (NJ, Estados Unidos), Transaction Publishers; traducción de M.A. González (2000): La relación entre la sociología y la filosofía. Madrid, EDAF.

CARRASCOSA, J.; FERNÁNDEZ, I.; GIL, D., y OROZCO, A. (1993): "Análisis de algunas visiones deformadas sobre la naturaleza de la ciencia y las características del trabajo científico", en: Enseñanza de las Ciencias, $\mathrm{n} . \stackrel{\circ}{ }$ extra (IV Congreso), 43-44.

CECJA (1992): Decreto 106/1992, de 9 de junio, por el que se establecen las enseñanzas correspondientes a la educación secundaria obligatoria en Andalucía. BOJA 56, Sevilla.

de PRO, A. (1995): “¿Formación de profesor de secundaria vs. profesor-tutor de prácticas de Secundaria?”, en L. Blanco y V. Mellado (eds.): La formación del profesorado de ciencias y matemáticas en España y Portugal, 375-397. Badajoz, Diputación Provincial.

DRIVER, R. y OLDHAM, V. (1986): "A constructivist approach to curriculum development in science. Studies in science education, 13, 105-122.

DUSCHL, R.A., y WRIGHT, E. (1989): "A case study of high school teachers' decision making models for planning and teaching science", en: Journal of Research in Science Teaching, 26(6), 467-501.

EFLIN, J.T.; GLENNAN, S., y REISCH, R. (1999): "The nature of science: a perspective from the philosophy of science", en: Journal of Research in Science Teaching, 36(1), 107-116.

FOUREZ, G. (1994): La construcción del conocimiento científico. Filosofía y ética de la ciencia. Madrid, Narcea.

GALLAGHER, J.J. (1991): "Prospective and practicing secondary school science teachers' knowledge and beliefs about the philosophy of science", en: Science Education, 75(1), 121-133.

GARCÍA-ESTAÑ, R.; de PRO, A., y VALCÁRCEL, M.V. (1999): "Un estudio de las concepciones de los licenciados en Biología y Química sobre la construcción del conocimiento c ientífico", en C. Martínez Losada, y S. García Barros (eds.): La didáctica de las ciencias. Tendencias actuales (XVIII Encuentros de Didáctica de las Ciencias Experimentales), 159-172. A Coruña, Servicio de Publicaciones da Universidad de la Coruña.

GORDON, D. (1984): "The image of science, technological consciousness, and the hidden curriculum”, en: Curriculum Inquiry, 14(4), 367-400. 
GUILBERT, L., y MELOCHE, D. (1993): "L'idée de science chez des enseignants en formation: Un lien entre I'histoire des sciences et l'hétérogénéité des visions?", en: Didaskalia, 2, 7-30.

HODSON, D. (1994): "Hacia un enfoque más crítico del trabajo de laboratorio", en: Enseñanza de las Ciencias, 12(3), 299- 313.

JIMÉNEZ, M.P. (1995): “Comparando teorías: la reflexión epistemológica sobre la naturaleza de la ciencia en la formación del profesorado", L.J. Blanco, y V. Mellado (eds.): La formación del profesorado de ciencias y matemáticas en España y Portugal, 267-280. Badajoz, Diputación Provincial.

KOULAIDIS, V., y OGBORN, J. (1989): "Philosophy of science: an empirical study of teachers' views", en: International Journal of Science Education, 11(2), 173-184.

KOULAIDIS, V., y OGBORN, J. (1995): "Science teachers' philosophical assumptions: how well do we understand them?", en: International Journal of Science Education, 17(3), 273-283.

KUHN, T.S. (1962): The structure of scientific revolutions. Chicago, University of Chicago Press; traducción de A. Contín (1971): La estructura de las revoluciones científicas. México D.F., Fondo de Cultura Económica.

LAKIN, S., y WELLINGTON, J. (1994): "Who will teach the "nature of science"?: Teachers' views of science and their implications for science education", en: International Journal of Science Education, 16(2), 175-190.

LEDERMAN, N.G. (1992): 'Student' and teachers' conceptions of the nature of science: a review of the research", en Journal of Research in Science Teaching, 29(4), 331-359.

LEDERMAN, N.G. (1999): "Teachers' understanding of the nature of science: factors that facilita te or impide the relationship", en: Journal of Research in Science Teaching, 36(8), 916-929.

LEDERMAN, N.G., y ABD-EL-KHALICK F. (1998): Avoiding de-natured science: activities that promote understandings of the nature of science, en W. McComas (ed.): The nature of science in science education: rationales and strategies, 83-126. Dordrecht, Kluwer Academic Publishers.

LLINARES, S. (1992): "Los mapas cognitivos como instrumento para investigar las creencias epistemológicas de los profesores”, en C. Marcelo (ed.): La investigación sobre la formación del profesorado. Métodos de investigación y análisis de datos, 57-95. Argentina, Cincel.

MANASSERO, M.A.; VÁZQUEZ, A., y ACEVEDO, J.A. (2001a): Avaluació dels temes de ciència, tecnologia $i$ societat. Palma de Mallorca, Consejería de Educación y Cultura del Gobierno de las Islas Baleares. 
MANASSERO, M.A.; VÁZQUEZ, A., y ACEVEDO, J.A. (2001b): "La evaluación de las actitudes CTS", en: Sala de Lecturas CTS+l de la OEI <http://www.campus-oei.org/salactsi/acevedo11.htm>. Versión en castellano del capítulo 2 del libro de M.A. Manassero; A. Vázquez, y J.A. Acevedo (2001a): Avaluació dels temes de ciència, tecnologia i societat publicado. Palma de Mallorca, Consejería de Educación y Cultura del Gobierno de les islas Baleares.

MARCELO, C. (1992): "Dar sentido a los datos: la combinación de perspectivas cualitativas y cuantitativas en el análisis de entrevistas", en C. Marcelo (ed.): La investigación sobre la formación del profesorado. Métodos de investigación y análisis de datos, 13-48. Argentina, Cincel.

MARCELO, C. (1994): "Estrategias de análisis de datos en investigación evaluativa”, en L.M. Villar (coord.): Manual de entrenamiento: evaluación de procesos y actividades educativas. Barcelona, PPU.

MATTHEWS, M.R. (1992): "Old wine in new bottles: a problem with constructivist epistemology, en: $\mathrm{H}$. Alexander (ed.): Philosophy of education 1992 (Proceedings of the Forty-Eighth Annual Meeting of the Philosophy of Education Society), 303-311. Urbana, Philosophy of Education Society. Versión en español (1994b): "Vino viejo en botellas nuevas: un problema con la epistemología constructivista", en: Enseñanza de las Ciencias, 12(1), 79-88.

MATTHEWS, M.R. (1994a): "Historia, filosofía y enseñanza de las ciencias: la aproximación actual”, en: Enseñanza de las Ciencias, 12(2), 255-277.

MATTHEWS, M.R. (1998): "The nature of science and science teaching”, en B.J. Fraser, y K.G. Tobin (eds.): International Handbook of Science Education, 981-999. Dordrecht, Kluwer Academic Publishers.

MEICHTRY, Y.J. (1993): "The impact of science curricula on student views about the nature of science", en: Journal of Research in Science Teaching, 30(5), 429-443.

MELLADO, V. (1995): “Concepciones de los profesores de ciencias en formación y práctica de aula”, en L. Blanco, y V. Mellado (eds.): La formación del profesorado de ciencias y matemáticas en España y Portugal309325. Badajoz, Diputación Provincial.

MELLADO, V. (1996): "Concepciones y prácticas de aula de profesores de ciencias, en formación inicial de primaria y secundaria", en: Enseñanza de las Ciencias, 14(3), 289-302.

MELLADO, V. (1997): "Preservice teachers' classroom practice and their conceptions of the nature of science", en: Science \& Education, 6, 331-354. También en B.J. Fraser, y K.G. Tobin (eds.) (1998): International Handbook of Science Education, 1093-1110. Dordrecht, Kluwer Academic Publishers.

MELLADO, V. (1998): “La investigación sobre el profesorado de ciencias experimentales”, en E. Banet, y A. de Pro (eds.): Investigación e innovación en la enseñanza de las ciencias, Vol I, 272 -283. Murcia, DM. 
MELLADO, V., y CARRACEDO, D. (1993): "Contribuciones de la filosofía de la ciencia a la didáctica de las ciencias", en: Enseñanza de las Ciencias, 11(3), 331-339.

MILES, M.B., y HUBERMAN, A.M. (1984): Qualitative data analysis: a soucerbook of new methods . Londres, Sage.

MONK, M., y OSBORNE, J. (1997): "Placing the history and philosophy of science on the curriculum: a model for the development of pedagogy", en: Science Educ ation, 81, 405-424.

MOSS, D.M.; ABRAMS, E.D., y ROBB, J. (2001): Examining student conceptions of the nature of science, en: International Journal of Science Education, 23(8), 771-790.

NRC (1996): National science education standards. Washington, DC, National Academic Press.

PALMQUIST, B.C., y FINLEY, F.N. (1997): "Preservice teachers' views of the nature of science during a postbaccalaureate science teaching program", en: Journal of Research in Science Teaching, 34(6), 595-615.

PALMQUIST, B.C., y FINLEY, F.N. (1998): A" response to Bell, Lederman, and Abd-El Khalick's explicit comments", en: Journal of Research in Science Teaching, 35(9), 1063-1064.

PETRUCCI, D., y DIBARURE, M.C. (2001): "Imagen de la ciencia en alumnos universitarios: una revisión y resultados", en: Enseñanza de las Ciencias, 19(2), 217-229.

PORLÁN, R. (1994): "Las concepciones epistemológicas de los profesores: el caso de los estudiantes de Magisterio", en: Investigación en la Escuela, 22, 67-84.

PORLÁN, R. (1995): “Las creencias pedagógicas y científicas de los profesores”, en Enseñanza de las Ciencias de la Tierra, 3(1), 67-84.

PORLÁN, R., y MARTíN DEL POZO, R. (1996): "Ciencia, profesores y enseñanza: unas relaciones complejas", en: Alambique, 8, 23-32.

PORLÁN, R., y RIVERO, A. (1998): El conocimiento de los profesores. Sevilla, Díada.

PORLÁN, R.; RIVERO, A., y MARTíN DEL POZO, R. (1998): "Conocimiento profesional y epistemología de los profesores, II: estudios empíricos y conclusiones”, en: Enseñanza de las Ciencias, 16(2), 271-288. 
REBOLLO, M. (1998): “Algunas visiones del profesorado de ciencias en formación inicial de secundaria sobre la naturaleza de la ciencia”, en E. Banet, y A. de Pro (eds.): Investigación e innovación en la enseñanza de las ciencias, Vol I, 294-303. Murcia, DM.

REID, D.J., y HODSON, D. (1989): Science for all. Londres, Cassell. Traducción de M.J. Martín-Díaz, y L.A. García - Lucía (1993): Ciencia para todos en secundaria. Madrid, Narcea.

RUBBA, P.A., y HARKNESS, W.L. (1993): "Examination of preservice and in-service secondary science teachers' beliefs about science-technology -society interactions", en: Science Education, 77(4), 407-431.

RUBBA, P.A.; SCHONEWEG-BRADFORD, C.S., y HARKNESS, W.J. (1996): "A new scoring procedure for the views on science-technology -society instrument", en: International Journal of Science Education, 18(4), 387-400.

RUGGIERI, R.; TARSITANI, C., y VICENTINI, M. (1993): "The images of science of teachers in Latin countries”, en: International Journal of Science Education, 15(4), 383-393.

RYDER, J., y LEACH, J. (1999): University science students' experiences of investigative project work and their images of science. International Journal of Science Education, 21, 945-946.

SOKAL, A., y, BRICMONT, J. (1998): Intellectual impos tures. Londres, Profile Books. Original en francés, 1997. Traducción de J.C. Guix Vilaplana (1999): Imposturas intelectuales. Barcelona, Paidós.

SUCHTING, W.A. (1992). Constructivism deconstructed. Science \& Education, 1, 223-254.

THOMAZ, M.F.; CRUZ, M.N.; MARTINS, I.P., y CACHAPUZ, A.F. (1996): "Concepciones de futuros profesores del primer ciclo de primaria sobre la naturaleza de la ciencia: contribuciones de la formación inicial”, en: Enseñanza de las Ciencias, 14(3), 315-322.

VÁZQUEZ, A. (1999): "Innovando la enseñanza de las ciencias: El movimiento ciencia-tecnología-sociedad", en: Revista del Colegi Oficial de Doctors i Llicenciats de Balears, 8, 25-35.

VÁZQUEZ, A.; ACEVEDO, J.A., y MANASSERO, M.A. (2000): "Progresos en la evaluación de actitudes relacionadas con la ciencia mediante el cuestionario de opiniones CTS", en I.P. Martins (coord.): OMovimento CTS na Península Ibérica. Seminário Ibérico sobre Ciência-Tecnologia- Sociedade no ensino-aprendizagem das ciencias experimentais, 219-230. Aveiro, Universidade de Aveiro. Versión electrónica corregida y actualizada en Sala de Lecturas CTS+l de la OEl. <http://www.campus-oei.org/salactsi/acevedo6.htm>, 2001.

VÁZQUEZ, A.; ACEVEDO, J.A., y MANASSERO, M.A. (2001): "Controversias y acuerdos sobre la nat uraleza de la ciencia: implicaciones para la enseñanza de las ciencias”, en Enseñanza de las Ciencias, no extra, tomo 2 (VI Congreso), 35-36. 
VÁZQUEZ, A.; ACEVEDO, J.A.; MANASSERO, M.A., y ACEVEDO, P. (2001): "Cuatro paradigmas básicos sobre la naturalez a de la ciencia”, en: Argumentos de Razón Técnica 4 (en prensa).

VÁZQUEZ, A., y MANASSERO, M.A. (1995a). Actitudes hacia la ciencia y sus relaciones con la tecnología y la sociedad en alumnos de todos los niveles educativos. Memoria de investigación. Madrid, MEC/CIDE.

VÁZQUEZ, A., y MANASSERO, M.A. (1995b): "Actitudes relacionadas con la ciencia: una revisión conceptual", en: Enseñanza de las ciencias, 13(3), 337-346.

VÁZQUEZ, A., y MANASSERO, M.A. (1997): Actitudes y valores relacionados con la ciencia la tecnologíay la sociedad en alumnado y profesorado. Implicaciones para la educación de las actitudes. Memoria de investigación. Madrid, MECD.

VÁZQUEZ, A., y MANASSERO, M.A. (1999a): "Evaluación educativa de los temas Ciencia-TecnologíaSociedad”, en C. Martínez Losada, y S. García Barros (eds): La didáctica de las ciencias. Tendencias actuales, 675-685. La Coruña, Servicio de Publicaciones de la Universidad de la Coruña.

VÁZQUEZ, A., y MANASSERO, M.A. (1999b): "Response and scoring models for the "Views on Science Technology- Society" instrument", en: International Journal of Science Education, 21(3), 231-247.

VÁZQUEZ, A.; MANASSERO, M.A., y ACEVEDO, J.A. (1998): "Modelos y cuestiones de evaluación en Ciencia, Tecnología y Sociedad (CTS)", en: Resúmenes de los XVIII Encuentros de Didáctica de las Ciencias Experimentales, 239- 240. : La Coruña. Servicio de Publicaciones de la Universidad de la Coruña.

VON GLASERSFELD, E. (1987): "An introduction to radical constructivism”, en: P. Watzlawick (ed.), The invented reality, 17-40. Nueva York, Norton. Versión española (traducción del original en alemán, 1981) de N.M. de Machain; I.S. de Luque, y A. Báez (1995): “Introducción al constructivismo radical”, en P. Watzlawick (ed.): La realidad inventada, 20-37. Barcelona, Gedisa.

VON GLASERSFELD, E. (1995):“Despedida de la objetividad”, en: P. Watzlawick, y Krieg, P. (eds.): Elojo del observador. Contribuciones al constructivismo. Barcelona, Gedisa, 19-31. [Original en alemán, 1991].

WOLPERT, L. (1992): The unnatural nature of science. Londres, Faber \& Faber Limited. Traducción de A. Linares (1994): La naturaleza no natural de la ciencia. Madrid, Acento Editorial. 


\title{
Contactar
}

Revista lberoamericana de Educación

\author{
Principal OEI
}

